\title{
VESTIBOLOGY
}

\section{Binocular cyclotorsion in superior vestibular neuritis}

\section{La ciclotorsione binoculare nella neurite vestibolare superiore}

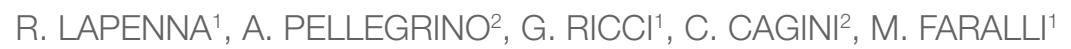 \\ Department of Surgical and Biomedical Sciences, ${ }^{1}$ Section of Otorhinolaryngology, ${ }^{2}$ Section of Ophthalmology, \\ University of Perugia, Italy
}

\begin{abstract}
SUMMARY
Conjugated cyclotorsion of the eyes toward the affected side can commonly be observed in vestibular neuritis. The aim of this study was to assess the differences in cyclotorsion between the ipsi- and contralesional eye during selective involvement of the superior branch of the vestibular nerve. We studied binocular cyclotorsion through ocular fundus photographs in 10 patients affected by acute superior vestibular neuritis (SVN). Cyclotorsion was also studied in 20 normal subjects. All SVN patients showed an ipsilesional cycloversion of the eyes. Normal subjects exhibited a constant mild excyclovergence $\left(6.42 \pm 2.34^{\circ}\right)$. In SVN patients, contralateral incyclotorsion $\left(8.4 \pm 8.14^{\circ}\right)$ was lower and not normally distributed compared to ipsilateral eye excyclotorsion $\left(17.9 \pm 4.36^{\circ}\right)$ with no correlation between them. The interocular difference in cyclodeviation could be related to the starting physiological excyclovergence, to different tonic effects on the extraocular muscles of the two eyes and to the different influence of spontaneous nystagmus on cyclodeviation in the two eyes. We recommend referring only to ipsilateral excyclotorsion in the evaluation of utricular function during SVN and its subsequent compensation. Further studies are required to determine the binocular cyclotorsion in the case of other kinds of selective involvement of the vestibular nerve.
\end{abstract}

KEY WORDS: Vestibular neuritis • Cyclotorsion • Utricle • Eye movement

\section{RIASSUNTO}

La ciclotorsione coniugata degli occhi verso il lato malato può comunemente essere osservata in corso di neurite vestibolare. Lo scopo di questo studio è stato quello di valutare le differenze nell'entità della ciclotorsione tra l'occhio ipsi- e contra- lesionale in caso di un coinvolgimento selettivo della branca superiore del nervo vestibolare. Abbiamo studiato la ciclotorsione binoculare ottenendo fotografie del fondo oculare in 10 pazienti affetti da neurite vestibolare superiore acuta (NVS). La ciclotorsione è stata studiata anche in 20 soggetti normali. Tutti i pazienti con NVS hanno mostrato una cicloversione ipsilesionale degli occhi. I soggetti normali invece hanno mostrato una exciclovergenza lieve costante $\left(6,42 \pm 2,34^{\circ}\right)$. Nei pazienti con NVS, l'inciclotorsione controlaterale $\left(8.4 \pm 8.14^{\circ}\right)$ era minore e non normalmente distribuita tra i soggetti rispetto alla exciclotorsione dell'occhio ipsilaterale $\left(17,9 \pm 4,36^{\circ}\right)$. Non è stata osservata correlazione significativa tra la ciclotorsione dei due occhi. La differenza interoculare della ciclotorsione potrebbe essere legato in parte alla presenza della exciclovergenza fisiologica, in parte ai diversi effetti tonici sui muscoli estrinseci dei due occhi, e infine alla diversa influenza del nistagmo spontaneo sulla ciclotorsione dei due occhi. Alla luce di questo studio consigliamo di far riferimento solo alla exciclotorsione ipsilaterale nella valutazione della funzione utricolare durante una NVS e del suo successivo compenso. Ulteriori studi sono necessari per determinare il comportamento della ciclotorsione binoculare in caso di coinvolgimento selettivo delle altre componenti del nervo vestibolare.

PAROLE CHIAVE: Neurite vestibolare $\bullet$ Ciclotorsione $\bullet$ Utricolo $\bullet$ Movimenti oculari

Acta Otorhinolaryngol Ital 2018;38:138-144

\section{Introduction}

Ocular cyclotorsion is defined as a rotation of the eye around its visual axis. Cyclotorsion can refer to the position of a single eye (in- or excyclotorsion or cyclodeviation) or even to the situation in both eyes (in- or excyclovergence or dextro- or levocycloversion). Cyclotorsion is absent when the vertical meridian traversing the centre of the cornea is parallel to a plumb line. In a state of incyclotorsion, the nasal end of the horizontal meridian is lower than the temporal part: the optic disk is lower than the fovea. Conversely, the optic disk is higher in cases of excyclotorsion ${ }^{1}$.

Torsional movements of the eyes are mainly determined by the inferior and superior oblique extraocular muscles. Cyclodeviations can be observed under those conditions that cause a functional imbalance between pairs of muscles inducing cyclotorsion, in the case of oblique axis astigmatism which is uncorrected or improperly correct- 
ed $^{2}$ and even as a complication following ocular or orbital surgery ${ }^{3}$.

Different methods have been proposed to measure cyclotorsion (scotometry, double Maddox rod test, cyclodeviation of the retinal vascular arcades) ${ }^{34}$, but fundus observation is considered to be the best method ${ }^{5}$.

Under normal conditions, cyclotorsion allows binocular single vision to be maintained when the head is tilted around the sagittal and/or transverse axis. This is guaranteed by a reflex of the extraocular muscles coming from activation of the vestibular receptors in response to the head motion. The torsional components of the vestibuloocular reflex (VOR) are mainly determined from activation of the ampullar receptors of the vertical semicircular canals and of the utricular macula ${ }^{6}$.

In the case of a sudden unilateral vestibular loss of function, a conjugated cyclotorsion of the eyes toward the affected side can be observed, together with alteration of vertical perception, skew deviation and head tilt of the eye-head postural reaction known as ocular tilt reaction (OTR), due to the acute vestibular asymmetry ${ }^{7}$. Except rare cases where an acute vestibular deficit is not followed by a lasting vestibular loss of function ${ }^{8}$, in vestibular neuritis the OTR may be long-lasting. While the altered perception of the subjective visual vertical ${ }^{9}$ represents an indirect sign of ocular cyclotorsion, and its improvement may depend on perceptual compensation due to neural plasticity following a vestibular deficit ${ }^{10}$, the evaluation of the ocular cyclotorsion can be used as a measure of long-term functional recovery after vestibular neuritis, depending directly on the utricular function ${ }^{11}$.

Few studies have focused on binocular cyclotorsion following acute vestibular asymmetry. Most have been conducted on patients who underwent a vestibular neurectomy for intractable Ménière's disease, and concluded that there was no significant difference in degrees between the excyclodeviated and incyclodeviated eyes during the acute phase following a complete vestibular nerve surgical resection ${ }^{12} 13$.

Even rarer are studies analysing the effect of acute vestibular asymmetry due to vestibular neuritis on binocular cyclotorsion ${ }^{11}{ }^{14}$, and to the best of our knowledge, there has been no publication discussing the possible different pathophysiological mechanisms acting on determination of cyclotorsion of the ipsi- and contralateral eye following acute vestibular loss.

As a consequence, it is not yet clear what is the best parameter to measure in the evaluation of cyclotorsion as a static utricular sign following a vestibular deficit, whether it is ipsilateral excyclotorsion, contralateral incyclotorsion, or the mean value of both.
Moreover, although it occurs more frequently in the region of the superior vestibular nerve (superior vestibular neuritis; SVN), vestibular neuritis could also affect the entire vestibular nerve (complete vestibular neuritis) or selectively only the inferior vestibular nerve (inferior vestibular neuritis) ${ }^{15} 16$.

Due to the involvement of different vestibular receptors, we hypothesise that, as with spontaneous nystagmus ( $\mathrm{SN}$ ), these three conditions should be considered separately in the study of binocular cyclotorsion of vestibular origin.

The aim of this work was to establish if there was a different behaviour in terms of degree excursion between ipsilateral excyclotorsion and contralateral incyclotorsion in response to acute unilateral SVN. A pathophysiological explanation of our findings is given.

\section{Materials and methods}

This was an observational case-control study based on a clinical population of 10 patients suffering from SVN and recruited during the period from January 2012 to December 2014. The study was conducted at the Department of Otolaryngology of the University of Perugia (Italy). The authors assert that all procedures contributing to this work comply with the ethical standards of the relevant national and institutional guidelines on human experimentation and with the Helsinki Declaration of 1975, as revised in 2008. Informed consent was obtained from all participants in the study.

All 10 patients received complete otoneurological, clinical and instrumental examination. Diagnosis of SVN was performed through meeting relevant criteria for vestibular neuritis proposed by Strupp and Brandt ${ }^{17}$. In particular, it was verified the presence of a pluripositional, unidirectional SN with well recognisable, dual horizontal and torsional components during its slow phase.

In order to verify the inferior vestibular nerve function, we recorded c-VEMPs (EPIC-Plus, LABAT, Italy) in all patients. We considered normal, symmetrical function when ${ }^{18}$ :

1. the P1N1 complex had a well recognisable morphology (between 13 and $23 \mathrm{msec}$ ) with a stimulus consisting of a click of $120 \mathrm{~dB}$ SPL;

2. P1N1 amplitude was between 50 and $70 \mathrm{nV}$;

3. the asymmetry ratio (AR), i.e. the ratio between the amplitude difference between the two sides (A1 and A2) and their sum, AR: (A1-A2) / (A1 + A2) X 100 where $\mathrm{A} 1>\mathrm{A} 2$, was lower than $35 \%$.

Patients with symptom onset longer than 3 days were excluded from the study. Those patients with a previous history of other otoneurological diseases were also excluded. MRI of the brain was conducted to exclude central lesions 
of any kind. Exclusion criteria also included a history of pre-existing abnormalities of eye movements, a history of ocular or orbital surgery, abnormal fundoscopic examinations and refractive defects.

At the same time as diagnosis of SVN, binocular cyclotorsion was measured by obtaining fundus photographs in both eyes taken in the sitting position with the head upright during fixation on a central target (SPECTRALIS $^{\circledR}$, Heidelberg Engineering, Heidelberg, Germany). The cyclodeviation angle was given by measuring the angle formed by a horizontal line passing through the fovea and a line which connects the centre of the fovea with the centre of the optical disc (Fig. 1). The final values of cyclotorsion for each eye were given by the mean of the angle obtained in five consecutive photographs to limit the effect of the torsional dynamic component of spontaneous nystagmus on static cyclotorsion ${ }^{19}$.

Cyclotorsion was measured with the same technique, but with a unique fundus photograph in both eyes in a group of 20 healthy volunteers, matched for age and sex.

\section{Statistical analysis}

The main parameter of the investigation was the amount in degrees $\left(^{\circ}\right)$ of the cyclotorsion measured in both eyes and expressed as positive values regardless of the direction. Average values were expressed as mean \pm SD.

The normality of the distribution of measured values in each data series was tested with the Shapiro-Wilk test, while Levene's test has been used to assess the homogeneity of variances among compared data sets. According to the results of the Shapiro-Wilk and Levene's tests, parametric paired $t$-test and non-parametric Wilcoxon signed rank test were used to compare paired data, while parametric Student's $t$ test and non-parametric Mann-Whitney $U$-test was used for unpaired data.

The correlation between excyclotorsion of the ipsilesional eye and incyclotorsion of the contralesional eye was studied with the non-parametric Spearman Rank-Order Correlation Coefficient.

Differences with $\mathrm{p}<0.05$ were considered to be statistically significant.

\section{Results}

\section{Control group}

All subjects showed some degree of excyclotorsion in both eyes (excyclovergence). Mean excyclotorsion was $6.89 \pm$ $2.21^{\circ}$ in the right eye and $5.95 \pm 3.42^{\circ}$ in the left eye.

Values of excyclotorsion (degrees) were normally distributed for $\mathrm{p}<0.05$ in both the right eyes ( $\mathrm{W}=0.90$; Shapiro-Wilk test), and the left eyes ( $\mathrm{W}=0.954$; Shapiro-Wilk test). Variances of excyclotorsion (degrees) of the right and left eyes were homogeneous $(\mathrm{a}=0.76$; Levene's test). There were no statistically significant differences in excyclotorsion between the right and left sides $(\mathrm{p}<0.05$; paired $t$-test). For this reason, the average value of the
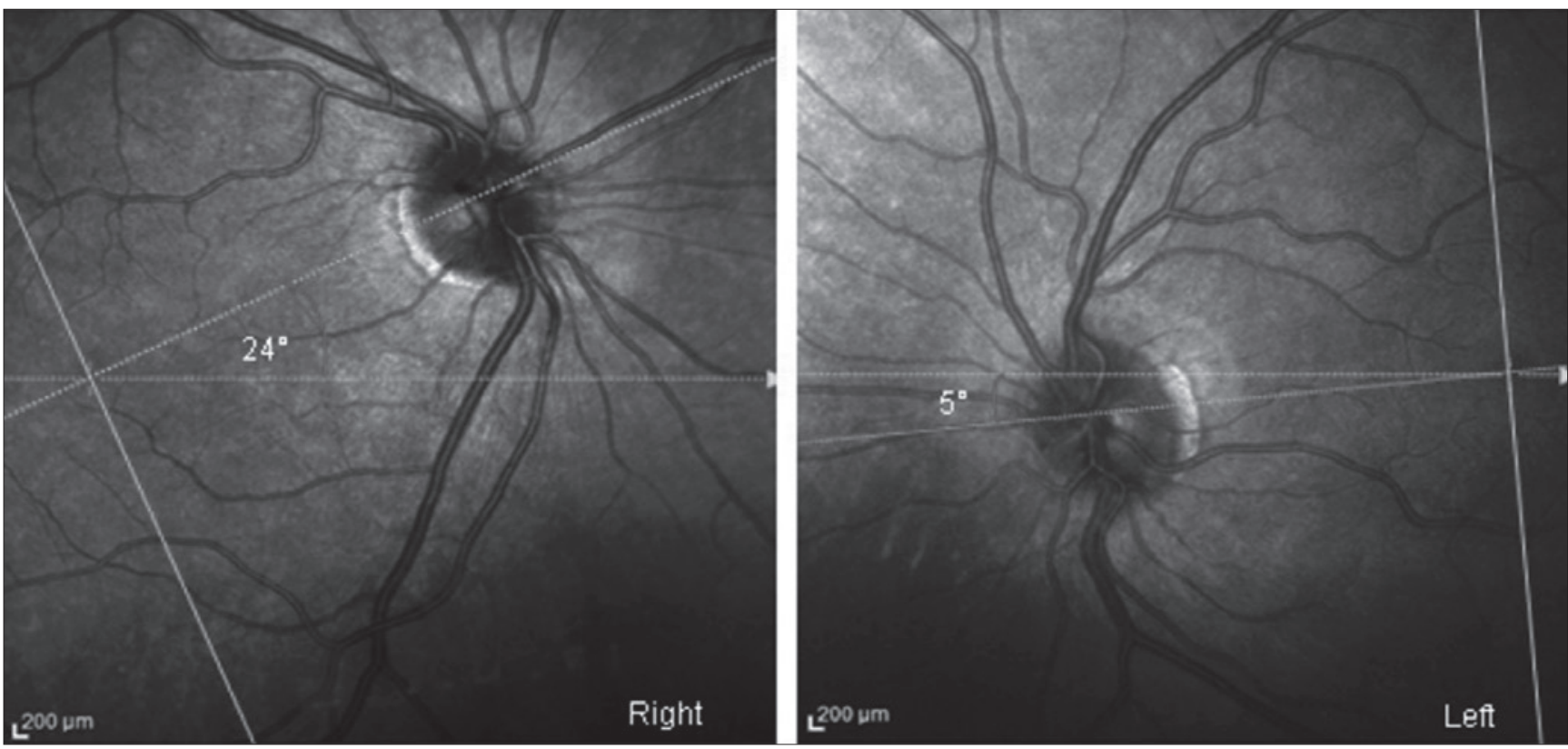

Fig. 1. Fundus photography in a patient with SVN (patient 6 from Table I) in the right eye. Excyclotorsion (right eye) and incyclotorsion (left eye) angles are shown. 
Table I. Cyclodeviation (minus sign before incyclotorsion values indicates the changing of the side of the deviation) in both eyes in patients during acute SVN and statistical analysis (Mann-Whitney U-test).

\begin{tabular}{lccccc} 
Patient & $\begin{array}{c}\text { Ipsilesional excyclotorsion } \\
\text { repeated measures }\end{array}$ & Mean \pm SD & $\begin{array}{c}\text { Contralesional incyclotorsion } \\
\text { repeated measures }\end{array}$ & Mean \pm SD & $\mathbf{p}$ \\
\hline 1 & $21 ; 19 ; 20 ; 21 ; 20$ & $20 \pm 0.84$ & $-10 ;-3 ;-4 ;-1 ;-1$ & $-4 \pm 3.70$ \\
2 & $16 ; 17 ; 18 ; 18 ; 16$ & $17 \pm 1$ & $-8 ;-6 ;-6 ; 1 ;-7$ & $-5 \pm 3.56$ & $-19 \pm 1.48$ \\
3 & $21 ; 20 ; 20 ; 20 ; 22$ & $21 \pm 0.89$ & $-20 ;-21 ;-19 ;-17 ;-19$ & $-9 \pm 3.70$ \\
4 & $15 ; 16 ; 15 ; 17 ; 16$ & $16 \pm 0.84$ & $-13 ;-11 ;-4 ;-10 ;-6$ & $-2 \pm 3$ & 0.000 \\
5 & $9 ; 7 ; 8 ; 10 ; 10$ & $9 \pm 1.3$ & $-1 ;-7 ;-1 ;-2 ;-1$ & 0.000 \\
6 & $24 ; 26 ; 25 ; 25 ; 24$ & $25 \pm 0.84$ & $-2 ;-5 ; 1 ; 1 ; 3$ & $-2 \pm 3.77$ \\
7 & $15 ; 16 ; 16 ; 17 ; 16$ & $16 \pm 0.71$ & $-3 ;-8 ;-2 ; 0 ; 2$ & $-21 \pm 1$ \\
8 & $18 ; 18 ; 19 ; 19 ; 19$ & $19 \pm 0.55$ & $-20 ;-22 ;-22 ;-21 ;-20$ & $-19 \pm 000$ \\
9 & $21 ; 22 ; 21 ; 21 ; 21$ & $21 \pm 0.45$ & $-21 ;-19 ;-20 ;-16 ;-20$ & 0.000 \\
10 & $15 ; 14 ; 16 ; 14 ; 16$ & $15 \pm 1$ & $-2 ;-8 ;-4 ; 0 ;-2$ & 0.000 \\
\hline
\end{tabular}

mean excyclotorsion of the two eyes in each patient was calculated $\left(6.42 \pm 2.34^{\circ}\right)$ with no significant difference between the mean values for the separate eyes and the average value of the mean excyclotorsion for the two eyes.

\section{SVN patients}

Ten patients ( 4 males and 6 females, mean age $42.8 \pm 8.35$ years) satisfied the inclusion criteria and were enrolled in the study.

Measurement of the five repeated photos of the ocular fundus of both eyes in each patient are reported in the Table I with the relative statistical analysis.

All patients, except patient 5 ( $\mathrm{p}=0.028$; Student's $t$ test), showed an excyclotorsion of the ipsilesional eye that was highly significantly greater compared to the normal range ( $\mathrm{p}=0.000 ;$ Student's $t$ test), while all patients showed incyclotorsion of the contralesional eye significantly different compared to the normal cyclotorsion $(\mathrm{p}=0.000$; Student's $t$ test).

In patient 5, the lower value of ipsilesional excyclotorsion can be explained supposing to start from a basal excyclotorsion of the lowest value of the normal range.

Mean ipsilesional excyclotorsion was $17.9 \pm 4.36^{\circ}$ with a normal distribution of the values at $\mathrm{p}<0.05(\mathrm{~W}=0.958$; Shapiro-Wilk test). Mean contralateral incyclotorsion was $8.4 \pm 8.14^{\circ}$ with a non-normal distribution of the values at $\mathrm{p}<0.05$ (W = 0.816; Shapiro-Wilk test).

Ipsilateral excyclotorsion was statistically significantly higher than the mean basal excyclotorsion in the control group ( $\mathrm{U}=2.5, \mathrm{p}<0.05$; Mann-Whitney $U$-test).

To assess the significance of the variation of mean contralateral incyclotorsion from the normal basal excyclotorsion (in degrees), their negative values were used so as to include the change in cyclotorsion direction in the statistical analysis, and the difference was statistically significant $(\mathrm{U}=0, \mathrm{p}<0.05$; Mann-Whitney $U$-test $)$. Variances between ipsilesional excyclotorsion and contralesional incyclotorsion were homogeneous $(\mathrm{a}=1.78$, Levene's test). The amount in degrees of the ipsilesional excyclotorsion was significantly higher than the contralesional incyclotorsion ( $\mathrm{Z}$-value $=-2.6, \mathrm{p}<0.05$; Wilcoxon signed rank test).

There was no significant correlation between the independent variable excyclotorsion and the independent variable incyclotorsion (Spearman Rank-Order Correlation Coefficient $=0.25 ; \mathrm{p}=0.48$ ), with a non-linear relationship between them.

\section{Discussion}

Cycloversion toward the affected side is consistently observed in unilateral vestibular loss. To the best of our knowledge, this is the first study in the literature focusing in detail on the differences between cyclotorsion of the ipsilesional and contralesional eye in vestibular neuritis, in particular, in the case of a lesion involving only the superior branch of the vestibular nerve.

Different physiological and pathological vestibular effects on the extraocular muscles should be considered in each eye.

Under static conditions, normal cyclotorsion is given by the synergic tonic action of the anterior semicircular canal (ASC), the posterior semicircular canal (PSC) and the medial part of the utricular macula (UM). The ASC activates the ipsilateral superior rectus muscle and the contralateral inferior oblique muscle. The PSC activates the contralateral inferior rectus muscle and the ipsilateral superior oblique muscle. The UM activates the ipsilateral superior rectus and superior oblique muscles and the contralateral inferior rectus and inferior oblique muscles (Fig. 2). 
In the case of SVN, we observed the simultaneous ipsilateral loss of function of both the ASC and the $\mathrm{UM}^{6}$.

As a consequence of deafferentation of the ASC, together with the consequent prevalence of the contralateral PSC, under static conditions, this will result in hypotropia of the ipsilesional eye and incyclotorsion of the contralesional eye ${ }^{6}$. As a consequence of deafferentation of the medial part of the UM, together with the prevalence of the contralateral UM, under static conditions, this will result in hypotropia and abnormal excyclotorsion of the ipsilesional eye, and hypertropia and incyclotorsion of the contralesional eye ${ }^{6}$.

It can be seen that an abnormal excyclotorsion of the ipsilesional eye is attributable only to UM involvement, while incyclotorsion of the contralesional eye is attributable to involvement of both the UM and ASC (Fig. 2B).

From the results of these tonic components, a consistently higher contralesional incyclotorsion than ipsilateral abnormal excyclotorsion should be expected.

This was not confirmed from our results which showed a significantly lower contralesional incyclotorsion. This could be partially explained by the basal physiological excyclotorsion that should exert an additive effect on ipsilesional abnormal excyclotorsion and a subtractive effect on contralesional incyclotorsion.

Figure 2 shows the expected changes from the normal excyclovergence in binocular cyclotorsion due to the asymmetrical activation of vestibular receptors in the course of a SVN (Fig. 2B), but also of inferior vestibular neuritis (Fig. 2C) or total vestibular neuritis (Fig. 2D). This model is, however, only theoretical. In fact, in our case, if the difference in degrees between ipsilateral abnormal excyclotorsion and contralateral incyclotorsion due to the onset of SVN was due only to the physiological basal bilateral excyclotorsion, they should be linearly correlated. This correlation is, however, not verified statistically (Spearman Rank-Order Correlation Coefficient $=0.25 ; \mathrm{p}=0.48$ ).

Therefore, an unpredictable external mechanism influencing the statistically significantly greater variability of the contralesional incyclotorsion than the ipsilesional abnormal excyclotorsion should be hypothesised. The reason for this may be explained in the potential effect of the biphasic ocular movement due to the concomitant $\mathrm{SN}$ on the tonic ocular torsional position.

The repetition in the single patient of the cyclotorsion measurement through five consecutive photos of both eyes showed a lesser dynamic effect of the SN in primary position on the ipsilesional excyclotorsion (low SD; Tab. I) than on the contralesional incyclotrosion (high SD; Tab. I).
In detail, $\mathrm{SN}$ can influence the value of cyclotorsion depending on during which of its phases, rapid or slow, the photograph is taken. The slow phase of the SN represents the compensatory phasic ocular movement that follows asymmetrical activation of the vestibular receptors in addition to its tonic effect. Otherwise, the rapid phase of the $\mathrm{SN}$ is anti-compensatory because it will tend to bring the eyes back to their initial position.

The contralesional incyclotorsion will be more pronounced as the fundus photograph is taken at the end of the slow phase of the SN. At this point, in fact, the eyes are found in their most extreme position toward the lesion side and the contralesional incyclotorsion will reach its peak value. On the other hand, if the fundus photograph is taken within the rapid phase of the $\mathrm{SN}$, the anti-compensatory phasic excyclotorsion will be opposed to the tonic incyclotorsion and the latter will be reduced even up to its potential suppression (at the end of the rapid phase).

Furthermore, the repetition of five consecutive photographs in each eye in order to minimize the dynamic effect of the $\mathrm{SN}$ is insufficient in reducing the interindividual variability of the contralesional incyclotorsion.

This may depend not only on possible differences in the tonic effect of the vestibular receptors involved, but also on the angular velocity of the SN. Theoretically, the faster the $\mathrm{SN}$, the higher the probability that the fundus photograph will be taken during the rapid phase of the SN and the lower will be the contralesional incyclotorsion and vice versa. However, further studies are required to correlate the angular velocity with the degree of contralesional incyclotorsion.

\section{Conclusions}

Although the tonic oculomotor component following SVN should be more pronounced in the contralesional eye, measurement of incyclotorsion showed lower and more inconsistent values than ipsilateral abnormal excyclotorsion. For this reason, due to its more constant behaviour and because it is a direct consequence of utricular deafferentation, we suggest that only ipsilesional abnormal excyclotorsion should be used for direct clinical evaluation of utricular dyfunction and for its subsequent compensation in case of SVN.

Future studies should investigate binocular cyclotorsion in other topographic localisations of vestibular neuritis. 
A
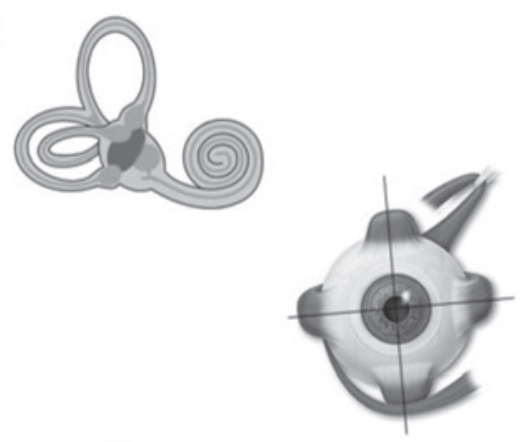

B
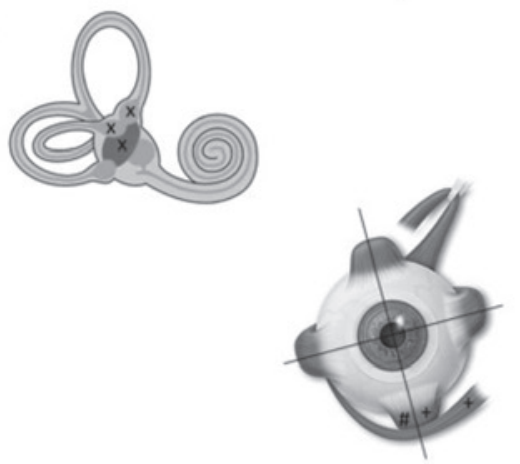

C
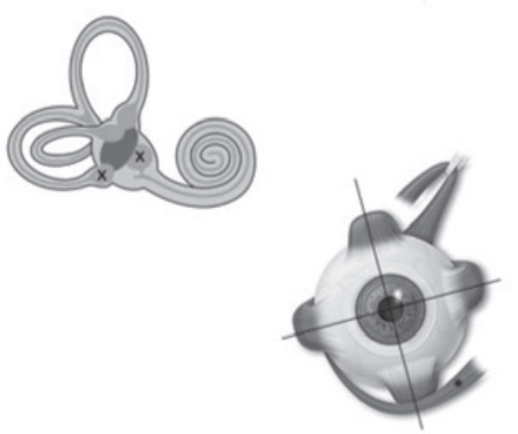

D
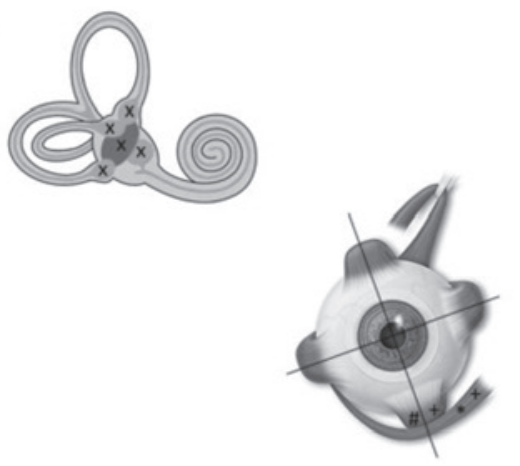
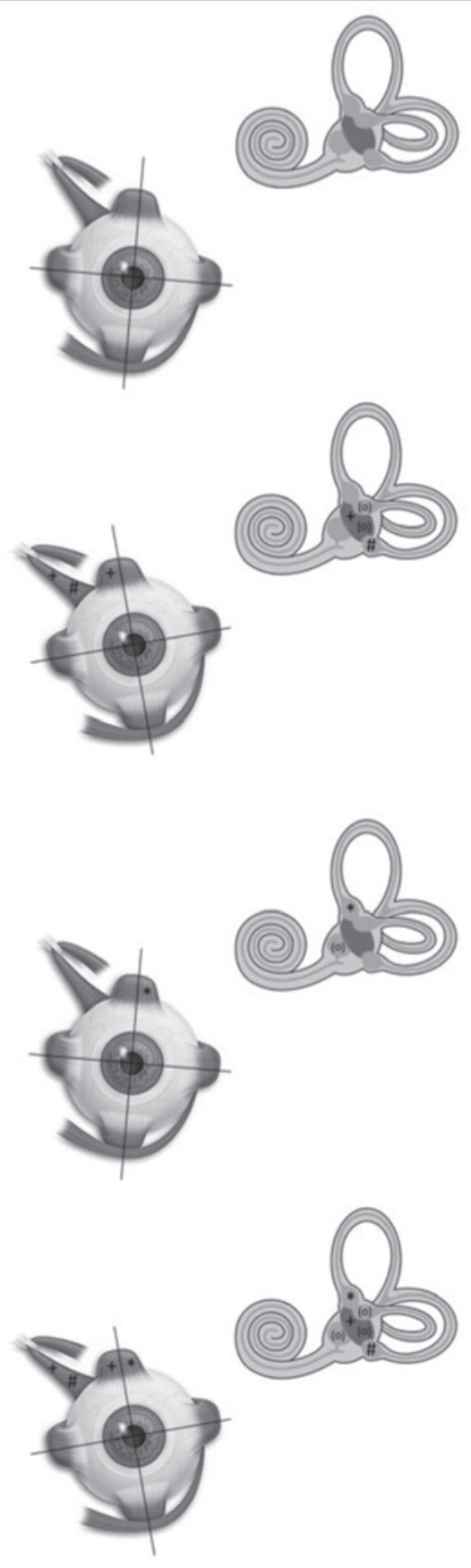

Fig. 2. Expected changes from the normal (A) in binocular cyclotorsion and vertical alignment due to the asymmetrical tonic activation of vestibular receptors. B: Right superior vestibular neuritis (SVN). Deafferentation (x) of the right anterior semicircular canal (ASC) and of the right utricular macula (UM). The left posterior semicircular canal (PSC) exerts a tonic effect (\#) on the left superior oblique muscle (SOM; incyclotorsion) and of the right inferior rectus muscle (IRM; hypotropia). The left medial part of UM exerts a tonic effect (+) on the left SOM, on the left superior rectus muscle (SRM; hypertropia), on the right inferior oblique muscle (IOM, excyclotorsion) and on the right IRM. The result will be a right hypotropia with an abnormal excyclotorsion, a left hypertropia with an incyclotorsion lower than the contralateral excyclotorsion. C: Right inferior vestibular neuritis (IVN). Deafferentation (x) of the right PSC and of the right saccular macula (SM). The left ASC exerts a tonic effect (*) on the left SRM and on the right IOM. The result will be an abnormal right excyclotorsion and a left hypertropia with a normal excyclotorsion. D: Right total vestibular neuritis. Deafferentation of all vestibular receptors (x). The tonic effect of the left PSC (\#), of the left medial part of the UM (+) and of the left ASC $\left(^{*}\right)$ is equivalent to the sum of the previous two situations. The result will be a right hypotropia with an abnormal excyclotorsion more pronounced than in SVN and IVN and a more pronounced left hypertropia than in SVN and IVN with an incyclotorsion comparable to that of SVN. The tonic effect $\left(^{\circ}\right.$ ) of the left horizontal semicircular canal, of the left lateral part of the UM and of the SM is not reported given their null effect on SOB, IOM, SRM and IRM. 


\section{References}

1 Spielmann A. Cyclotorsions. J Fr Ophtalmol 2002;25:959-67.

2 Toselli C, Miglior M. Oftalmologia Clinica. Bologna: Monduzzi Editore; 1979.

3 Lemos J, Eggenberger E. Clinical utility and assessment of cyclodeviation. Curr Opin Ophthalmol 2013;24:558-65.

4 Parsa CF, Kumar AB. Cyclodeviation of the retinal vascular arcades: an accessory sign of ocular torsion. Br J Ophthalmol 2013;97:126-9.

5 Seo JM, Kim K, Kim JH, et al. Measurement of ocular torsion using digital fundus image. Proceedings of the $26^{\text {th }}$ Annual International Conference of the IEEE EMBS. San Francisco, CA, USA, September 1-5, 2004.

6 Marcelli V. Vestibologia clinica. Casi clinici e test diagnostici. Pavia: Selecta Editrice; 2013.

7 Halmagy GM, Gresty MA, Gibson WP. Ocular tilt reaction with peripheral vestibular lesions. Ann Neurol 1979;6:80-3.

8 Loader B, Linauer I, Korkesch S, et al. A connection between neurovascular conflicts within the cerebellopontine angle and vestibular neuritis, a case controlled cohort study. Acta Otorhinolaryngol Ital 2016;36:421-7.

9 Faralli M, Lapenna R, Mandalà M, et al. The first attack of Ménière's Disease: a study through SVV perception. Clinical and pathogenetic implications. J Vestib Res 2014;24:335-42.

10 Micarelli A, Chiaravalloti A, Schillaci O, et al. Aspects of cerebral plasticity related to clinical features in acute vestibular neuritis: a "starting point" review from neuroimaging studies. Acta Otorhinolaryngol Ital 2016;36:75-84.

11 Strupp M, Arbusow V, Maag KP, et al. Vestibular exercises improve central vestibulospinal compensation after vestibular neuritis. Neurology 1998;51:838-44.

12 Devèze A, Montava M, Lopez C, et al. Vestibular compensation following vestibular neurotomy. Eur Ann Otorhinolaryngol Head Neck Dis 2015;132:197-203.

13 Lopez C, Borel L, Magnan J, et al. Torsional optokinetic nystagmus after unilateral vestibular loss: asymmetry and compensation. Brain 2005;128:1511-24.

14 Kim HA, Hong JH, Lee H, et al. Otolith dysfunction in vestibular neuritis: recovery pattern and a predictor of symptom recovery. Neurology 2008;70:449-53.

15 Goebel JA, O’Mara W, Gianoli G. Anatomic considerations in vestibular neuritis. Otol Neurotol 2001;22:512-8.

16 Kim JS, Kim HJ. Inferior vestibular neuritis. J Neurol 2012;259:1553-60.

17 Strupp M, Brandt T. Vestibular neuritis. Semin Neurol 2009;29:509-19.

18 Welgampola MS, Colebatch JG. Vestibulocollic reflexes: normal values and the effect of age. Clin Neurophysiol 2001;112:1971-9.

19 Babić B. Cyclotropia after unilateral vestibular damage. Srp Arh Celok Lek 130 Suppl 2002; 1:1-7. [Article in Serbian]. 